\section{Consecuencias políticas de los conflictos socio-territoriales. Hacia una conceptualización pertinente*}

\section{Political consequences of socio-territorial conflicts. Toward an adequate conceptualization}

\author{
Gonzalo Delamaza *
}

\footnotetext{
Agradezco los comentarios al texto realizados durante las presentaciones de versiones iniciales del mismo en el Seminario "Conflictos Socio Territoriales en América Latina" (Lisboa 2017); LASA y REPAL (Lima 2017). Asimismo, los aportes de mis colegas Maritza Paredes, Antoine Maillet y Christian Martínez. El artículo forma parte de los resultados del proyecto Fondecyt 1180496. El autor agradece el apoyo financiero de FONDECYT.
}

** CEDER Universidad de Los Lagos. República 517 Santiago. gonzalo.delamaza@ulagos.cl

\section{Resumen}

Se propone una conceptualización pertinente acerca de cómo los conflictos socio territoriales están produciendo consecuencias políticas, en la región andina de América del Sur, para hacer posible la investigación comparada. Se analiza el campo político institucional, el de las políticas públicas y de las relaciones de poder entre los actores del conflicto en dos ámbitos: dentro del territorio (la escala local) y más allá de él (diversas escalas). Se examinan casos a partir de fuentes secundarias y se discuten las metodologías utilizadas para construir bases de datos. Los actores del conflicto son más diversos y con menor peso organizativo que los movimientos sociales, pero al mismo tiempo ejercen roles de mediación, anteriormente cumplidos por organizaciones formales como los partidos políticos. Las consecuencias no surgen de un conflicto en particular, sino principalmente de su acumulación y combinación con elementos del entorno. Surgen nuevas formas de politización: nuevos actores, en lugares no tradicionales y en ausencia de los mediadores tradicionales. El paso de lo local a lo nacional y a lo internacional no opera como escala progresiva, sino como una dinámica multiescalar y socio institucional, que se comprende mejor con la noción de conflicto que con la de movimientos sociales.

Palabras clave: Conflictos locales; movimiento social; extractivismo; politización; Región Andina
Abstract
Our aim is to propose a relevant conceptualization about how socio-territorial conflicts are 
producing political consequences, based on evidence from countries of the Andean Region, to make possible comparative research. The political institutional field, public policies and power relations between the actors of the conflict in two areas -within the territory (local scale) and beyond the territory (several scales) - are analyzed. Cases are examined from secondary sources and methodologies used to build databases are discussed. Conflict actors are more diverse and present less organizational weight than traditional social movements but, at the same time, they exercise mediation roles, previously fulfilled by formal organizations such as political parties. Consequences do not arise from a particular conflict, but mainly from its accumulation and combination with elements of the political environment. New forms of politicization around these conflicts emerge: new actors in non-traditional places and in the absence of traditional political mediators. The shift from the local to the national and the international does not operate as a progressive scale, but as a multi-scale and socio-institutional dynamic, which is better understood with the notion of conflict than with social movements.

Key words: Local conflicts; social movement; extractivism; politicization; Andean Region

\section{Los conflictos socio-territoriales y sus consecuencias}

El auge del extractivismo y la economía basada en los recursos naturales produjo un período de acelerado crecimiento económico, pero al mismo tiempo acarreó profundas transformaciones en losterritorios-especialmente rurales-de América Latina y multiplicó los conflictos originados en los territorios (Haarstadt 2012; Svampa 2013). Una proporción importante de dichos conflictos han girado en torno a la minería, los hidrocarburos y la energía (Bebbington y Bury 2013; Delamaza et al. 2017; Li 2015; Quiroga et al. 2012; Ulloa y Coronado 2016) y el agua (Panfichi y Coronel 2011; Boelens et al. 2011). Pero también a partir de ello, se han producido conflictos en relación al ordenamiento político de las regiones y sus competencias (Penaglia et al. 2016; Quiroga et al. 2012). Es relevante observar diferente tipo de actores sociales, diferentes lugares -muchas veces apartados de los grandes centros urbanos- y la emergencia de nuevos temas que han aparecido en el panorama social y político de la región. Esto contribuye a caracterizar problemáticas actuales, parcialmente diferentes de las prevalentes durante el largo período del Estado desarrollista, así como también de la desarticulación que acompañó a la implantación del neoliberalismo en la región (Calderón, 2012). Más allá del eventual incremento de eventos de protesta, la extensión de estos conflictos por amplias zonas de cada país, su continuidad en el tiempo, la amplitud de los actores concernidos en ellas, la diversidad de escalas territoriales en que algunos de ellos se despliegan, así como la variedad y significación de los problemas en cuestión para los territorios, permiten considerarlos como una forma de acción colectiva importante en sí misma. Con similitudes respecto del concepto de movimientos sociales, se trata, sin embargo, de conflictos que tienen también especificidades que identificaremos, puesto que ello es relevante para al objeto específico de este trabajo: las consecuencias políticas de los conflictos.

El texto analiza consecuencias tanto en lo que toca a las modificaciones institucionales y la implementación de políticas públicas, como a los cambios en las relaciones de poder entre los 
actores de los mismos territorios donde surgen los conflictos. Incorpora ejemplos empíricos de cuatro países de la región, con el objetivo de desarrollar una conceptualización útil para una visión agregada de las consecuencias políticas de la conflictividad local. Analizar la manera en que las transformaciones de la acción colectiva surgidas del contexto señalado influyen sobre los resultados de la misma, permitirá enriquecer un campo de investigación relativamente menos estudiado, así como proponer agendas de investigación futura.

Bajo la denominación de political outcomes of social movements se ha desarrollado una literatura académica relativamente reciente y ciertamente de menor desarrollo que la dedicada a los motivos de surgimiento, dinámicas, actores, framing y repertorios de acción de los movimientos sociales (Amenta 2014; Amenta et al. 2010; Bosi et al. 2016; Kolb 2005). Esta literatura está caracterizada por varios aspectos, que a la vez establecen sus límites. En primer término su universo de referencia principal son los llamados movimientos sociales, cuya corriente principal (Tarrow 2011) incorpora las dimensiones de interacción, conflicto e identidad compartida. Se apunta así a identificar la constitución de un sujeto de la acción colectiva que la sostiene y reproduce y a examinar las relaciones de ese sujeto con otros. Por ello se estudia el "movimiento feminista", el "movimiento estudiantil", el "movimiento antiglobalización", etc. De tal manera que o bien se trata de: a) categorías sociales que irrumpen en el panorama de la acción colectiva constituyendo nuevos sujetos de acción colectiva, anteriormente invisibilizados o desarticulados, b) de "causas" que unifican a los diversos componentes del movimiento.
En el tipo de conflictos que este artículo trata, en cambio, el origen es territorialmente muy diverso, en base a causas muy específicas. Por tanto, en principio son dinámicas fragmentadas una de otra, que no necesariamente se estructuran como movimientos sociales en sentido tradicional, aunque eventualmente comparten algunas de sus características. Así, por ejemplo, Silva (2016) analiza el impacto de la Campaña "Patagonia sin Represas" en la región de Aysén en el sur de Chile, con algunos instrumentos de la teoría de movimientos sociales, utilizados en conjunto con otros instrumentos provenientes de la ecología política. Pérez (2012) analiza a su vez el "movimiento social de Aysén", para referirse al período de movilizaciones de febrero de 2012, lo que corresponde más bien a una etapa de manifestaciones masivas en el desarrollo de un conflicto de mayor duración. Un conflicto que se relaciona con la campaña mencionada y que a la vez es más amplio que ella, originando sus propios resultados. Panfichi y Coronel (2011) analizan los "conflictos hídricos" en el Perú, distinguiendo aquellos que se acercan más específicamente a la categoría de movimiento social. Penaglia et al. 2016 categorizan las diferentes orientaciones de los movimientos regionalistas en Chile, buscando patrones de convergencia y a la vez de diferenciación entre ellos.

Asumiendo la diversidad de origen y conformación, preferimos mantener la denominación de conflictos socio-territoriales, que entendemos primariamente conformados por redes asimétricas de actores, algunos de los cuales se constituyen en torno al conflicto mismo, redes normalmente vinculadas de acuerdo a una geometría variable, que incluye en ocasiones vínculos institucionales más o menos estables (principalmente con las 
autoridades locales y también con funcionarios que se consideran a sí mismos como parte de la movilización social). ¿Cómo es que fenómenos netamente locales logran impactos políticos que los sobrepasan, en ausencia de las instituciones políticas mediadoras usuales?

En la teoría de los movimientos sociales, normalmente se distingue entre consecuencias internas y externas. La dimensión política está incorporada entre las externas, lo cual se desprende de la consideración de los movimientos sociales como "sujetos" actuando en un "entorno político" determinado. Al enfocarse en los conflictos socio-territoriales es posible incorporar una dimensión de politización que incluye también los procesos políticos que surgen en los propios territorios a consecuencia de los conflictos, donde resulta más difícil distinguir entre lo interno y lo externo. Cuando se trata de cambios electorales o nuevos actores del juego político local, la distinción resulta relativamente sencilla: un movimiento determinado se ve asociado a la emergencia de nuevos líderes políticos, a la promoción de cambios en la composición de los apoyos electorales o al surgimiento de un nuevo actor. Pero si el propio movimiento es el que se convierte en un actor con influencia política, incluso en el ámbito nacional, la categoría se desdibuja un tanto. Lo que está en juego aquí es la propia noción del sujeto de la acción. Donoso y Von Bülow (2017) han puesto el énfasis en la diversidad interna de los movimientos, donde también se encuentran actores institucionales, que ya no son sólo considerados como "entorno", "aliados" u "opositores" a la dinámica del movimiento.

Adicionalmente lo usual es que el análisis de las consecuencias políticas se limite a la institucionalidad y las políticas públicas (Giugni et al. 2013). En relación a esta última dimensión los conflictos locales pueden contribuir a impactar en una dimensión local de la política, que no necesariamente se proyecta en el ámbito de la política nacional de modo directo. Como veremos, cuando se trata de los resultados, las escalas y los niveles se entrecruzan, sin necesariamente evolucionar de la misma manera en las diferentes escalas.

Por último, contamos con poca teorización a partir de casos y contextos latinoamericanos. Trabajos muy recientes abordan el tema en la región, incorporando el análisis de consecuencias, a la vez que flexibilizando la noción misma de movimientos sociales. Podemos mencionar aquí los trabajos de Silva (2015, 2016, en prensa), el volumen sobre Chile de Donoso y Von Bülow (2017) y el de Tatagiba y Teixeira (2017) que retoma críticamente el modelo de efectos combinados.

La hipótesis general del trabajo es que en los conflictos socio-territoriales de la región andina, se aprecia el surgimiento tanto nuevo actores políticos como de nuevas formas de influencia política: en distintos lugares (no urbanos, periféricos), mayoritariamente distantes de los actores tradicionales de la política (partidos y organizaciones formales) y a través de procesos de organización y movilización diversos y heterogéneos (redes de articulación, sin constituir necesariamente movimientos sociales, con orientaciones diferentes entre sí). En el caso de Bolivia una perspectiva histórica más larga permite apreciar con claridad las diferencias entre el movimiento sindical de los mineros y la revolución campesina de 1952 y la politización actual de los conflictos. De una parte la movilización que antecedió al triunfo 
del Movimiento al Socialismo (MAS) y, sobre todo, la dinámica que se generó a partir de allí, con un gobierno que se entiende como estrechamente ligado a los movimientos sociales. En el caso de Perú, se requeriría un análisis histórico más profundo para establecer similitudes y diferencias sistemáticas entre los movimientos campesinos que se opusieron a la minería en los años cincuenta y sesenta y los actuales conflictos. Aun así las consecuencias institucionales y políticas que se observan actualmente (política indígena, liderazgos políticos regionales y otros) no se habían producido anteriormente. En el caso de Chile, el modelo extractivista se impuso hace varios decenios y la conflictividad en torno a él se aprecia a partir del año 2005 aproximadamente. Colombia, por último, introduce la minería como prioridad de la política económica muy recientemente y ello parece estar detrás de esta conflictividad.

La evidencia empírica surge de diferentes fuentes existentes en la Región Andina, donde el extractivismo es más pronunciado. En el caso de Chile, se elaboró un catastro de conflictos (http://cispo.ulagos.cl/wp/), mientras para Perú se utiliza la información proveniente de los reportes periódicos elaborados por la Defensoría del Pueblo y un interesante desarrollo de trabajos de investigación que utilizan esa fuente y la elaboran (http://www. defensoria.gob.pe/temas.php?des=3), así como la agregación regional desarrollada por Moisés Arce en la Universidad de Missouri (http://faculty.missouri.edu/_arcem). Aunque en menor medida, también se incorporan datos de Bolivia y Colombia, principalmente a partir de fuentes secundarias y de estudios de caso (Quiroga et al., 2012; Ulloa y Coronado 2016). Se utilizan como apoyo otros catastros de conflictos (INDH 2016; OCMAL 2015). Vale decir, en el artículo no hay un tratamiento sistemático de los datos empíricos agregados, difícilmente comparables. Más bien se utiliza la evidencia y los casos como ejemplos de la pertinencia de la conceptualización propuesta.

Es necesario hacer una precisión sobre el alcance del análisis propuesto. La denominación "socioterritorial" puede incluir conceptualmente los conflictos urbanos (defensa de barrios, conflictos con la especulación inmobiliaria, contaminación, demandas por servicios), pero nos parece que, operacionalmente es necesario distinguirlos de los que aquí analizamos, pues de otro modo la diversidad se incrementa en extremo. Por otra parte seleccionamos los conflictos alejados de los centros metropolitanos, puesto que allí es más nítido observar la dinámica del paso de lo local a la politización, que es el centro del análisis.

Por razones análogas hemos excluido el análisis de los movimientos indígenas como tales, puesto que requieren de su propio enfoque. Solo incluimos aquí los conflictos locales que involucran la participación de actores indígenas. ${ }^{1}$ La siguiente sección aborda la discusión sobre acción colectiva, conflictos y movimientos sociales y establece nuestra conceptualización sobre conflictos socio-territoriales. En la sección 3 se propone una categorización operacional para el estudio de las consecuencias de los conflictos socio-territoriales. En la cuarta y quinta partes se discuten los resultados relativos a la dimensión político institucional y a las políticas públicas, respectivamente. En la sexta parte se presentan los resultados relativos a las consecuencias sobre los territorios, los propios

Sobre aspectos operacionales ver Delamaza et al. 2017 
movimientos y la acción colectiva. Finalmente se formulan conclusiones sobre cómo abordar este fenómeno y se proponen algunos temas para investigación futura.

\section{Acción colectiva, conflictos y movimientos sociales}

Lo novedoso del proceso de politización que se advierte en los conflictos socio-territoriales es el tipo de tránsito que efectúan hacia lo político. Inicialmente en muchos casos podría tratarse simplemente de asuntos que atañen a un grupo particular en función de intereses también particulares, similares a los descritos en la literatura de los denominados conflictos tipo "nimby's" (not in my backyard). Los conflictos en torno a la ubicación de una determinada inversión pública, en defensa de una reserva natural o contra las externalidades negativas de una planta industrial, muchas veces se inician de este modo. Y, posiblemente, muchos conflictos permanecen en esa situación de mayor o menor confrontación o resolución por vía judicial, mediación u otra. Sin embargo, también muchos de ellos trascienden esa situación inicial, iniciándose así un proceso de politización de la acción colectiva. Así, por ejemplo, lo sugiere la misma duración y evolución de los conflictos y la variedad de actores que en él se involucran, que rebasan a los directamente involucrados. También los repertorios que utilizan, propios más bien de acción organizada y destinada a suscitar la atención pública y las respuestas políticas.

Un ejemplo de lo anterior es la movilización de vecinos de la nortina ciudad de Arica en Chile por relocalización de sus viviendas surgió como una demanda de atención en salud, pues los niños y ancianos enfermaban mucho, sin causa aparente. No fue el problema existente - hasta ese momento desconocido- el que motivó el conflicto, más bien fue la actividad conflictiva la que permitió movilizar tanto al municipio, como a la universidad local y las autoridades de salud para establecer que los problemas de salud se debían al alto contenido de plomo en el terreno donde las viviendas estaban emplazadas. Hasta la actualidad se disputa sobre compensaciones, relocalización y remoción del plomo. Pero al mismo tiempo el conflicto suscitó una respuesta institucional local a través de la creación de una oficina municipal de medio ambiente y un significativo conflicto a nivel nacional luego de su difusión pública a través de un canal de televisión en un programa de denuncia. Junto a los intentos de solución locales, se modificó la norma chilena de plomo permitido en lugares habitados (Arriagada 2012). ${ }^{2}$

En el caso de Tambogrande en Perú, la oposición a la instalación de una mina en un valle de productores agrícolas no dio espacio a compensaciones económicas individuales como planteara la empresa, sino que a un proceso que pasó por una consulta popular en la localidad, manifestaciones en el centro de Lima durante el funcionamiento de las instancias institucionales de decisión y una eficaz campaña de propaganda y sensibilización para volver

\footnotetext{
Los desechos habían sido depositados por parte de una empresa sueca en 1985, las viviendas se construyeron durante los años noventa, el conflicto surgió a comienzos de los años 2000 y ha durado hasta la actualidad, combinando denuncia, protesta, acciones de mitigación, respuestas de la autoridad, cambios legales y surgimiento de nuevas entidades comunitarias (Arriagada 2012). En 2019 en el contexto de la realización en Chile de una importante conferencia ambiental (COP 25) las organizaciones sociales concernidas han invitado a la activista sueca Greta Thunberg a visitar Arica. El conflicto se va moviendo entre lo local, lo nacional y lo internacional recursivamente.
} 
"nacional" un problema "local". ${ }^{3}$ La efectividad de la movilización llevó a otras experiencias, en Perú y otros países, a utilizar métodos similares para amplificar sus demandas, así como a la consagración posterior del derecho a consulta en el Perú (Arce 2015; De Echave et al. 2009; Li 2015)

Pero, ¿cómo conceptualizar esta emergente acción colectiva en los territorios? Si no es puramente reacción defensiva que se satisface con una respuesta específica o se apaga con una acción represiva, ¿qué es? No responde fácilmente a la conceptualización tradicional de movimiento social (Diani 2015). De hecho en el caso peruano al menos una analista indica que estos conflictos no logran constituirse como tales en el ámbito nacional (Paredes 2015) y otro análisis indica que ello está fuertemente influido por el contexto político cambiante que ha existido en Perú (Panfichi y Coronel 2015). Tampoco en Chile o Colombia se han estructurado organizaciones nacionales significativas o estrategias coherentes en torno a las cuales se desenvuelvan las acciones. De acuerdo a Giugni "contention crystallizes into a social movement when it taps embedded social networks and connective structures and produces collective action frames and supportive identities able to sustain contention with powerful opponents" (2004: 148). Al poner el énfasis en la acción performativa, de acuerdo a esta definición, muchos conflictos locales cumplirían las condiciones mínimas para ser considerados como un movimiento social. Sin embargo la conceptualización sobre movimientos sociales también agrega que la identidad colectiva debe ser compartida, a

La campaña vinculaba el interés de los productores de limones de Tambogrande con el pisco sour, connotada como la "bebida nacional" peruana. pesar de que los lazos sean informales tanto en los movimientos sociales como en este tipo de conflictos (Diani 2015). Si bien es teóricamente posible que estos conflictos deriven en un movimiento social, al parecer su fragmentación territorial y el carácter localizado de sus luchas sugieren modalidades parcialmente distintas de constitución.

En definitiva lo más relevante para diferenciar este tipo de acción colectiva es el hecho que esta se constituye "en el conflicto", sin que los actores, el repertorio, las orientaciones, etc. necesariamente preexistan a él. Vale decir, se trata de un fenómeno que evoluciona con el conflicto mismo y en esa evolución va generando sus propios dispositivos de acción. En el caso de Chile se verifica un importante número de "coordinadoras" y "asambleas ciudadanas", definidas en función del problema territorial existente (Valenzuela 2015). Lo mismo ocurre en Colombia y Perú, donde adoptan nombres como "frente de defensa de". La denominación misma sugiere el carácter no institucionalizado de los actores, aunque la duración de las organizaciones puede prolongarse por largo tiempo, variando según los casos. A la vez que es también contingente a los eventos emergentes, tanto del propio territorio como del entorno de oportunidades y amenazas existentes. Por ello puede evolucionar organizacionalmente, asimismo como también en términos de orientaciones y repertorios de acción. Diani (2015: 11) ha introducido matices en esta dirección, al revisar el concepto de movimientos sociales, insertándolo en una tipología de formas de coordinación de la acción colectiva. Eso le permite plantear que la acción colectiva también puede constituirse en coaliciones de actores, donde los vínculos entre estos tienen un carácter más instrumental y cambiante en el 
tiempo. Nos parece que esta idea de "coalición de actores" resulta más ajustada a la realidad de este tipo de conflictos.

Lo anterior permite no "suponer" la existencia de un movimiento social a partir de una serie de eventos conflictivos, que bien pueden también estar articulados en función de objetivos instrumentales, variables en el tiempo, etc. Pero además permite subrayar tanto la composición como las formas de acción plurales que es frecuente encontrar en estos conflictos. La convergencia de actores locales movilizados por un problema concreto con actores de la institucionalidad pública sensibles al problema por diversas razones (boundary agents) y dispuestos a actuar en determinados campos que les son propios, como aliados de los actores locales, es un ejemplo de ello. Pero también lo es otro tipo de alianzas más amplias como las que se establecen con las ONG -algunas de ellas internacionales y con agendas globalesque se movilizan en función de esos conflictos, en la medida que perciben la vinculación con las causas y estrategias más amplias en las que están insertas. Las coaliciones que se forman entre estos y otros actores no necesariamente nacen de una coincidencia completa de intereses entre ellos, sino de momentos en los cuales los objetivos de cada uno de ellos coincide, al menos parcialmente con los de los demás. Evidentemente estas coaliciones evolucionan, favorecen la "contaminación" o difusión entre territorios diversos, así como la difusión de ideas y debate entre objetivos y estrategias a perseguir en cada momento. De esa manera la coalición se reconfigura y evoluciona a lo largo del tiempo y cada uno de los actores ocupa lugares y roles diferentes en esa evolución. Pero la evolución es también contingente a los eventos emergentes, tanto del propio territorio como del entorno de oportunidades y amenazas existentes. Por ello puede evolucionar organizacionalmente, así como también en términos de orientaciones y repertorios de acción.

Pero ¿cuándo estamos en presencia de un conflicto de este tipo? Vinculando entre sí los argumentos anteriormente expuestos, se puede decir que conceptualmente los conflictos socioterritoriales, como objeto de análisis, se sitúan entre los eventos de protesta y los movimientos sociales. Mientras estos dos últimos centran su interés en los actores movilizados (y sus oponentes), el estudio de las consecuencias resulta especialmente relevante para un enfoque de conflictos, pues es el desenlace de aquellos. En el caso de este trabajo lo haremos específicamente en torno a las consecuencias políticas, uno de los aspectos más controvertidos y difíciles de conceptualizar, como veremos más adelante.

Los conflictos los hemos conceptualizado en su doble condición de "locales" y "políticos". En relación al carácter local, este supone varias condiciones para cumplirse. Estas condiciones son necesarias, pues en sentido general todo conflicto y toda acción sucede en un territorio. Lo que interesa aquí son aquellos que al mismo tiempo se refieren a asuntos del territorio y son protagonizados por actores locales. De acuerdo a esto, las condiciones que hacen al carácter local del conflictos son: a) que emerge de problemas que afectan directamente a los habitantes del territorio (normalmente no surgen en virtud de una "causa" o "ideología", que bien puede estar presente, sino en virtud de un problema concreto o percibido como tal que afecta a una localidad o zona); b) incluye acción contenciosa en el mismo territorio o protagonizada por los actores del territorio. 
Como veremos luego, los conflictos son locales, pero no puramente locales cuando se politizan; c) las demandas involucran territorios específicos o incluyen asuntos que tienen consecuencias directas sobre el territorio. Ello puede ser tan concreto como el cierre de una faena industrial, el monitoreo de las externalidades ambientales de una mina, el reparto de regalías provenientes de las ganancias o tan amplio como el estatuto de autonomía para una región determinada.

El otro componente es, evidentemente, la vía de politización del conflicto. En este caso no se trata de varias condiciones que deben cumplirse, sino que apenas una de ellas es suficiente, puesto que el proceso de politización puede producirse de diferentes maneras. En primer término puede deberse a los actores del conflicto, en la medida que otros actores, ajenos a los que originalmente fueron portadores del motivo inicial y principal del conflicto, se involucran en él. Las razones de ello pueden ser diversas -solidaridad, vínculos, proximidad territorial, estar viviendo conflictos similares- pero el involucramiento de actores diversos está mostrando ya un plus con respecto a la situación inicial. Una segunda vía de politización son las demandas involucradas. Las consideramos de este modo cuando estas no se dirigen únicamente-y a veces no se dirigen del todo- hacia los responsables directos del problema que está en el origen, sino que apelan al Estado de diversas formas. Es usual que lo interpelen en su función normativa, regulatoria o sancionatoria, aunque a veces también lo hacen en busca de mediación y compensaciones. Finalmente la politización se puede expresar en el framing del conflicto por parte de los actores del mismo. Vale decir cuando se ponen en debate orientaciones de la política pública, como ocurre con descentralización, medio ambiente, regulación de la inversión extranjera, derechos sobre recursos del territorio, etc. ${ }^{4}$

Un aspecto relevante en relación a este tipo de conflictos es su multiescalaridad, vale decir el hecho que junto a estar anclados y sucediendo en un territorio específico, comprometen asuntos que trascienden ese territorio, incluyendo muchas veces un componente internacional. Esto afecta a los actores y también al tipo de consecuencias que surgen de la conflictividad. Este vínculo local / internacional se produce por varios motivos. En primer lugar porque las problemáticas sobre la cual los conflictos surgen muchas ocasiones involucran la presencia de empresas multinacionales, porque afectan el comercio internacional de un país o un producto altamente sensible internacionalmente (como el petróleo y los hidrocarburos en general), fluctuando según sea esa sensibilidad en diferentes momentos (los precios suben y bajan permanentemente). En segundo término porque en el escenario internacional actual, algunas decisiones están sancionadas por tratados de libre comercio, sujetas a regulaciones negociadas en el espacio internacional e incluso la resolución de diferencias confiado a instancias situadas fuera de las fronteras nacionales y, por lo tanto, excluidas del juego político nacional. Por otra parte las agendas internacionales en diversos temas son muy influyentes en los países, cuando no directamente impuestas como condicionantes de fondos de cooperación, etc. Desde la protección a la propiedad intelectual y las patentes farmacéuticas, a la igualdad

Kunrath et al. (2017) discuten con mucho detalle las ventajas y limitaciones de la aplicación de las categorías de framing y sus distintos componentes y variantes, a la acción colectiva en el caso de los movimientos sociales. Para el caso de Chile ver también la conceptualización de Aguilera y Alvarez (2017). 
de género o incluso los derechos indígenas vienen avalados por esa agenda internacional y por esa vía se ha inducido su incorporación en políticas nacionales. Todo lo anterior lleva a que la participación de actores que se mueven en la escala internacional sea muy frecuente en algunos de estos conflictos. $Y$ lo interesante es que esto no solo se reduce a los organismos internacionales que avalan el modelo extractivista y lo promueven en sus políticas, sino también en la contestación a los mismos, a través de ONG, redes de organizaciones, etc., que también actúan en el nivel global (Von Bülow 2010; Paredes 2016; Urkidi y Walter 2011). Las coaliciones se reconfiguran y evolucionan a lo largo del tiempo y cada uno de los actores con recursos y escalas de movilización e incidencia asimétrica ocupan lugares y roles diferentes a la articulación móvil de la coalición (Fraser 2007).

Vistos los antecedentes anteriores, se explica, al menos parcialmente, la tendencia de conflictos que en apariencia son estrictamente locales, a trascender esa escala y politizarse de diversas formas.

\section{Como abordar las consecuencias de los conflictos socio-territoriales}

¿Cómo se vinculan los rasgos anteriores a las consecuencias políticas que surgen del tipo de conflictos que hemos conceptualizado? Estas consecuencias pueden darse en el territorio mismo donde sucedió el conflicto o bien alcanzar otras escalas, como la política nacional o sectorial, cambios en el campo jurídico y normativo. En el ámbito territorial identificamos al menos cuatro dimensiones en que estas consecuencias pueden ocurrir. Por una parte, a través del surgimiento de nuevos actores que no existían antes del conflicto (o reconfiguración de los actores preexistentes) o bien por la modificación de las relaciones de poder entre los actores del territorio. Por otra, en un terreno más institucional, esto puede expresarse en un cambio de orientaciones del gobierno local (cambio político) o bien en la creación de nuevas instituciones, surgidas para enfrentar de diversas formas el problema expresado en el conflicto. Más allá del territorio también se pueden verificar cambios de orientación o regulación de las políticas públicas en algún sector específico como ocurre con medio ambiente, minería, etc. O bien a través de la creación de instituciones más o menos participativas luego de los conflictos. Por último puede verificarse la modalidad tradicional de mediación política, cual es que la demanda sea incluida por los candidatos o partidos en sus plataformas electorales. ${ }^{5}$

Antes de desarrollar las distinciones mencionadas, sinteticemos las categorías de análisis expuestas en el siguiente diagrama:

\footnotetext{
No hemos incluido aquí las respuestas inmediatas que los gobiernos entregan a los conflictos en busca de asegurar la gobernabilidad, la política de "apagar incendios", relacionada más bien con temas de orden público, puesto que es justamente la premura de los gobiernos por "terminar el conflicto" la que muchas veces impide artificialmente su procesamiento político (Paredes 2015).
} 


\begin{tabular}{|c|c|c|}
\hline $\begin{array}{l}\text { Carácter Socio-Territorial } \\
\text { del Conflicto Local } \\
\text { (condiciones necesarias) }\end{array}$ & $\begin{array}{c}\text { Vías de Politización del } \\
\text { Conflicto (una condición es } \\
\text { suficiente) }\end{array}$ & $\begin{array}{l}\text { Consecuencias políticas } \\
\text { posibles del conflicto }\end{array}$ \\
\hline $\begin{array}{l}\text { Problema local: } \\
\text { Emerge de problemas que } \\
\text { afectan directamente a los } \\
\text { habitantes del territorio }\end{array}$ & $\begin{array}{l}\text { Actores: } \\
\text { Otros actores se involucran } \\
\text { en el conflicto }\end{array}$ & $\begin{array}{l}\text { En el territorio: } \\
\text { Cambia el mapa de actores } \\
\text { sociales }\end{array}$ \\
\hline $\begin{array}{l}\text { Movilización local: } \\
\text { Se produce movilización en el } \\
\text { territorio }\end{array}$ & $\begin{array}{l}\text { Demandas: } \\
\text { van más allá del conflictos } \\
\text { específico (una empresa, un } \\
\text { barrio) apelando al Estado }\end{array}$ & $\begin{array}{l}\text { Cambian las relaciones de } \\
\text { poder } \\
\text { Se modifican orientaciones } \\
\text { del gobierno local } \\
\text { Se crean nuevas instituciones }\end{array}$ \\
\hline $\begin{array}{l}\text { Demanda territorial: } \\
\text { Las demandas involucran } \\
\text { territorios específicos }\end{array}$ & $\begin{array}{l}\text { Framing: } \\
\text { Se ponen en debate } \\
\text { orientaciones de la política } \\
\text { pública (descentralización, } \\
\text { medio ambiente, regulación } \\
\text { de la inversión extranjera, } \\
\text { derechos sobre recursos del } \\
\text { territorio, etc.) }\end{array}$ & $\begin{array}{l}\text { Más allá del territorio: } \\
\text { Se crean nuevas instituciones } \\
\text { o se modifican antiguas } \\
\text { Se modifican políticas o } \\
\text { normativas } \\
\text { Surgen nuevas fuerzas } \\
\text { políticas } \\
\text { Actores políticos incluyen } \\
\text { demandas en sus programas } \\
\text { y plataformas* }\end{array}$ \\
\hline
\end{tabular}

* Esta sería la modalidad tradicional de mediación política por eso no se incluye en la operacionalización Fuente: elaboración propia

Al igual como ocurre con los movimientos sociales, las consecuencias e impactos pueden ser de carácter directo, indirecto o mixto (Giugni 2004) y a su vez pueden ser de carácter progresivo -en términos de apertura e inclusividad de la política pública o de incremento del peso de los actores movilizados en el territorio- o bien regresivo, si el impacto corre en sentido contrario a lo que se demanda, como ocurre con la represión, por ejemplo. También nos parece importante establecer una distinción temporal de las consecuencias, las que pueden ser inmediatas o de corto plazo y también de largo plazo. Finalmente, las consecuencias han sido conceptualizadas como influidas por las estrategias y capacidades de los actores (la 
teoría de la movilización de recursos) y también en función de las oportunidades políticas (teoría del proceso político). Parece razonable incorporar ambas dimensiones, en lo que se ha denominado un modelo de efectos acumulativos y combinados, donde las consecuencias de la acción colectiva se producen en combinación con elementos del contexto (Tatagiba y Teixeira 2016). Evidentemente las consecuencias pueden ser difícilmente atribuibles a un conflicto en particular y entenderse mejor como un "efecto acumulativo" de varios de ellos (Silva 2016) ya sea en un mismo territorio o por la reiteración de conflictos en torno a causas similares en diferentes lugares; así como de "efecto combinado" entre los recursos movilizados y las oportunidades políticas (Tatagiba y Teixeira 2016). Todas estas distinciones ayudan a ir más allá de la noción de "éxito vs. fracaso" de los conflictos o de sus "fortalezas y debilidades". Esas categorías suponen un sujeto preexistente y evalúan la efectividad de su acción normalmente según criterios normativos no siempre explícitos. Un enfoque de conflicto permite un despliegue diferente, donde prima la diversidad de actores y el juego diferenciado de los distintos ámbitos, temporalidades, profundidad y orientación de las consecuencias.

Sin duda que un estudio detallado de los diversos conflictos locales arrojaría una gran variabilidad de resultados en las dimensiones seleccionadas y al mismo tiempo resultaría una tarea de gran envergadura y dificultad. La literatura sobre el tema, además, indica que en gran medida los resultados políticos de la movilización son contingentes al contexto en el cual suceden, a saber, el régimen político, la estructura de oportunidades, entre otros (Panfichi y Coronel 2015; Delamaza et al. 2011). En este trabajo hemos privilegiado una visión general que nos permita apreciar la posible utilidad del marco conceptual y metodológico. En el estado actual de la investigación empírica ello nos conducirá necesariamente a hacer alusión a estudios de caso, sin que podamos por ahora contar con datos agregados de manera sistemática y comparable para todos los países considerados. Por ello este apartado debe considerarse solo como una aproximación. En las secciones siguientes abordamos primero las consecuencias fuera del territorio, para luego analizar las que se verifican en los territorios mismos.

\section{Consecuencias en el ámbito político nacional}

En relación a las consecuencias políticas de tipo institucional, una primera dimensión de cambio posible podría ser el cambio del régimen político. Esta dimensión normalmente se incluye entre las consecuencias políticas posibles de la acción de los movimientos sociales (Bosi et al. 2016). Sin embargo, al tratarse de conflictos locales, es difícil que alcancen este impacto. Aunque podría considerarse que la emergencia y ascenso al poder de Evo Morales y el MAS en Bolivia, se relaciona en cierto modo a una expansión de conflictos locales contenciosos, desde los años noventa, hasta producirse la aparición de nuevos actores políticos y la presión por el cambio de régimen. Sería el caso que podría ejemplificar un vínculo en el cual se enlaza la acción política local con el cambio a nivel nacional. ${ }^{6}$ Sin embargo, el paso

\footnotetext{
La base de apoyo de Evo Morales tuvo un fuerte componente territorial en el sur andino y en el sector cocalero originado por el desplazamiento desde la minería de Potosí. En cambio en las regiones de la llamada "media luna", ese apoyo fue mucho menor. Dentro de una definición ideológica general de izquierda, también los elementos territoriales tenían relevancia.
} 
a lo político estuvo menos determinado por la dinámica territorial que por la crisis política del Estado boliviano.

Pero la vinculación entre movimientos políticos territoriales y el gobierno del MAS tuvo consecuencias posteriores, sobre todo a partir del segundo período de gobierno de Evo Morales. En la medida que el Estado se ha fortalecido y la participación de los movimientos que adhieren al gobierno está institucionalizada -al punto que el propio Morales sigue siendo presidente de la Federación de Cocalerosla emergencia de nuevas movilizaciones no encuadradas en ese esquema se vuelve políticamente conflictiva. El caso más visible es el producido en la zona amazónica del Tipnis, por el proyecto gubernamental de construcción de una carretera transamazónica que atraviesa la reserva natural y territorios indígenas (Barros 2013). La dinámica del conflicto se parece en gran medida a otras de la región, como Tambogrande, pero difiere en sus resultados, puesto que la coalición opuesta a la carretera no logra detener la iniciativa gubernamental. Ello ocurre a pesar de que la movilización coincide con el momento de mayor visibilidad internacional del discurso político del "buen vivir" y el reconocimiento de los derechos indígenas en Bolivia (De la Fuente 2017). Vale decir, predomina el esquema tradicional de mediación y control político.

En Colombia, luego de un período de fuerte expansión de la frontera minera avalada por una definición gubernamental de la minería como "locomotora económica" del país, se sucedieron múltiples conflictos en diversas localidades con baja presencia previa de la minería. La utilización de consultas populares por parte de autoridades locales llevó a una contienda de competencias con el ejecutivo. En un fallo de 2014, la Corte Constitucional establece que las autoridades del nivel nacional deberán acordar con autoridades territoriales medidas para protección del ambiente y el licenciamiento, aplicando principios de coordinación, concurrencia y subsidiaridad. Al mismo tiempo se avaló el derecho de las autoridades locales a realizar consultas sobre temas mineros. Este fallo obligó al gobierno a elaborar un Plan Nacional de Ordenamiento Minero con participación de comunidades locales (González 2017). La particularidad del caso es la intervención de la Corte Constitucional como reguladora del conflicto político-institucional, lo que da una proyección nacional a los conflictos locales. No puede considerarse que ello altere al sistema político, pero modifica las condiciones en que se procesa la actividad minera y las iniciativas locales.

Lo usual es que los cambios que se producen como consecuencia, al menos parcial, de los conflictos socio-territoriales no alcancen una envergadura política global, afectando a los modelos de desarrollo. En Chile, Silva (2016) analiza el caso de la Campaña "Patagonia sin Represas" contra la instalación de centrales hidroeléctricas en el extremo sur del país (Hydroaysén), que tuvo un enorme éxito en detener un mega proyecto que contaba con apoyo internacional y del Estado chileno. A pesar de ello, según al autor ni ese movimiento ni los demás han logrado modificar el panorama general de la política energética ni el modelo extractivista que demanda la realización de proyectos como Hydroaysén. De acuerdo a este autor, ello dependería de los "efectos acumulativos de las luchas locales" (Silva, 2016: 19), un aspecto escasamente estudiado, que concordamos en su importancia. En el caso de 
Perú, Maritza Paredes indica que los conflictos en torno a la minería se "glocalizan", lo cual, en el plano nacional implica que "logran poner en la agenda nacional temas controversiales importantes", como el derecho a la consulta o la imparcialidad de una autoridad ambiental, pero sin construir nuevas estructuras convergentes de identidad política y organizacional que trasciendan el nivel local y que puedan asegurar un efectivo seguimiento y negociación de esas agendas" (Paredes, 2009: 138).

\section{Las consecuencias sobre las políticas públicas}

El análisis anterior no implica que los conflictos no tengan un impacto sobre las políticas públicas, tanto en su orientación general (agenda setting) como en aspectos sectoriales, nuevos mecanismos más inclusivos, etc. La literatura centrada en la crítica global del extractivismo considera a éste como un fenómeno de larga duración, proveniente desde el período colonial y prácticamente inalterado desde entonces. Por ello tiende a subestimar este tipo de logros o, en el mejor de los casos, considerarlos como estrategias destinadas a contener o manipular los procesos de "resistencia" de las comunidades (Roa y Navas 2014). Sin embargo desde el punto de vista de las comunidades afectadas $\mathrm{o}$ de la evolución de la agenda política, se pueden advertir transformaciones significativas en las agendas y prácticas de las políticas públicas que luego generan también diversos tipos de respuesta desde las localidades. Un caso específico es la ya mencionada consagración de la consulta previa, libre e informada en relación a los territorios habitados por comunidades indígenas, pero que ha tenido también otras aplicaciones más amplias. El derecho a consulta, basado en el Convenio 169 de la OIT fue ratificado por Colombia desde hace ya unos veinte años y no puede considerarse propiamente un resultado de los conflictos territoriales, que más bien se intensificaron durante el período de aplicación del convenio. En el caso de Perú y Chile, en cambio, su ratificación es mucho más reciente. En Perú como parte de una nueva política hacia los conflictos relacionados con la minería del presidente Ollanta Humala (2011 - 2016) en oposición a su antecesor Alan García (2006 2011) (Arce 2015: 207). Fue la crisis detonada por los sucesos violentos ocurridos en el Baguá en la selva amazónica en 2009 , la que movilizó una respuesta política que modificó las reglas del juego (Panfichi y Coronel 2015).

Un caso específico de impacto sobre la normativa nacional, que incluso requería modificaciones constitucionales, y que nació y se desarrolló directamente a partir de una movilización territorial indígena es el de la Ley de Espacio Marítimo Costero para los Pueblos Originarios, aprobaba en 2007 en Chile. Dicha ley, conocida como "ley lafkenche" establece la prevalencia de los derechos indígenas sobre el territorio marítimo (o "maritorio") a aquellas comunidades que puedan demostrar uso consuetudinario de los recursos del mar (Delamaza y Flores 2012). Fue impulsada y negociada directamente por la organización Identidad Territorial Lafkenche, que agrupa a las comunidades indígenas de la costa de Tirúa, al sur del Golfo de Arauco, en la región del Bío Bío. Aquí el componente de movilización contenciosa es menos relevante que la fortaleza organizacional, el uso estratégico de las oportunidades políticas y la conquista de apoyos transversales en los decisores políticos (Delamaza et al. 2011) 
Otro caso que es posible mencionar en Chile es el de la política energética formulada entre 2014 y 2016. A pesar que en el estudio sobre Patagonia sin Represas ya citado se señala que "despite these positive outcomes, the Patagonia Defense Council was not able to alter Chile's general policy towards large-scale hydropower generation" (Silva 2016: 18), lo cierto es que la actual política reduce significativamente el peso de las grandes centrales, en beneficio de las minicentrales o centrales de pasada y el incremento de la producción de energías renovables (Maillet 2017). ${ }^{7}$ La reformulación política incluyó también una modificación institucional en el Ministerio de Energía, por medio de la creación de una División de Participación y Diálogo Social y la puesta en práctica de diversos mecanismos de participación para la elaboración de la política y la realización de proyectos en el área. ${ }^{8}$

El problema que se plantea en los casos anteriores es, sin embargo, el de la atribuibilidad de los cambios de política como impacto de los conflictos, dada la multidimensionalidad del fenómeno y la multicausalidad que es esperable en relación a una reforma de envergadura en cualquier ámbito de política. ¿Hasta dónde se puede afirmar que una reforma es consecuencia

También se ha estudiado el impacto de la movilización producida en contra de la destrucción de glaciares por parte del proyecto binacional Pascua Lama (de la minera Barrick Gold) en el norte de Chile, en la discusión legislativa de la Ley de Protección de Glaciares (Cortez 2016). Si bien es posible establecer el nexo, principalmente en la presentación del proyecto de ley, la tramitación legislativa parece haber omitido la gran mayoría de los asuntos que interesaban a los habitantes movilizados en contra de la intervención de los glaciares en Pascua Lama.

8 La "División" es el nivel jerárquico superior de la burocracia ministerial, dependiente directamente de la Subsecretaría correspondiente. Sobre las modificaciones en el ministerio ver: http://www.energia.gob.cl/que-entendemos-por-participacionciudadana de uno o más conflictos? Igual pregunta se plantea a los estudiosos de los movimientos sociales a la hora de abordar las consecuencias de estos. Resulta difícil establecer causalidad, por lo que la estrategia de investigación debe orientarse hacia la determinación de correlaciones significativas, intentando aislar factores recurrentes en diferentes situaciones. Por ejemplo, en el caso de las grandes centrales de energía en Chile, al parecer el cambio coincide con una reorientación de las empresas hacia proyectos de menor envergadura por distintas consideraciones de viabilidad (Maillet 2017). Evidentemente una de ellas es la conflictividad existente a partir de las mayores externalidades de ese tipo de proyectos. Pero también existen otras consideraciones de tipo tecnológico, económico y de mercado que forman parte de esta reformulación. Dada la diferencia de escala entre los conflictos socio-territoriales y las políticas e instituciones nacionales, es esperable una situación como esta. El desafío pasa a ser, entonces, más que atribuir una causalidad singular a un conflicto, intentar establecer el rol específico que uno o más conflictos (su "efecto acumulativo") puede tener sobre determinadas decisiones de política.

En la literatura es posible encontrar varias distinciones útiles para entender este "efecto acumulativo". Entre ellas la referida a las diferentes áreas como a las fases de la política pública, el papel de las redes con participación de agentes institucionales y/o actores políticos y la intervención de la variable internacional en las decisiones. En relación a las fases de la política en las que conflictos de este tipo pueden tener mayores impactos, la evidencia muestra que estos tienden a ser mayores en las etapas de formación de agenda, puesto 
que los conflictos serían protagonizados principalmente por actores no institucionales o provenientes de fuera del sistema político y porque los medios utilizados les permitirían hacer aparecer públicamente los problemas, llamar la atención de las autoridades y demandar soluciones. Pero para que la demanda ingrese en la fase decisional y de implementación de las políticas requiere algún grado de involucración de los agentes políticos tradicionales y de la burocracia pública. Estos eventualmente se movilizarán como reacción al conflicto, aunque recibirán también la presión de las elites políticas y de sus propios intereses. Al mismo tiempo necesitarán traducir en propuestas institucionales y políticas viables, lo cual requiere tanto de una voluntad política que respalde, como de ideas, lenguajes y otros instrumentos propios de ese campo.

Un ejemplo de lo anterior es el de la reforma a la ley minera en El Salvador. Bebbington (2016) observa que en el caso de los temas relativos a acceso a recursos naturales, estos resultan de mucha mayor dificultad para transformarse que las políticas ambientales. Dado que los primeros se relacionan con el modelo de acumulación sustentado en acuerdos políticos globales (en muchos casos avalados además por acuerdos internacionales), una modificación sustentable en el tiempo probablemente estará vinculada a cambios en los acuerdos políticos de fondo. Esto explicaría también el hecho que en ocasiones se verifiquen avances y luego retrocesos, lo cual obliga a cualificar el tipo de impacto o resultado de un conflicto. Vale decir este puede afectar una o más políticas en el corto plazo pero no de manera sustentable o bien lograr una modificación que abre un nuevo ciclo en determinadas áreas de política (Ibíd.). La reflexión aplica también a la ya descrita tramitación del Convenio 169 de la OIT en el caso chileno. ${ }^{9}$

El punto anterior reafirma la importancia de considerar los "efectos combinados" entre las dinámicas territoriales y fenómenos políticos globales. ${ }^{10}$ Esto obliga a reflexionar sobre la economía política de los conflictos y diferenciar entre diversas áreas de política y sus respectivas dificultades y oportunidades. No es sólo que la estructura de oportunidades políticas varía en el tiempo, dando mayor o menor posibilidad de impacto a determinados conflictos. Es también que las políticas públicas están más o menos incrustadas (embbedded) con los modelos de desarrollo y los arreglos políticos globales.

La pluralidad de actores involucrada en los conflictos y el carácter de las coaliciones, que muchas veces incluyen actores institucionales, así como vínculos a redes internacionales es un factor a considerar en el impacto que pueden tener dichos conflictos, particularmente respecto de la "transmisión" o "contaminación" que se produce entre diversos conflictos, en cuanto a la efectividad de determinadas estrategias y el aprendizaje colectivo, tal como se mencionó en el caso de Tambogrande y el

El proyecto se presentó a comienzos de los años noventa, como parte de los acuerdos de la transición democrática, y aunque los conflictos impulsaron el debate, las características de la reforma legal de 2008 que lo ratificó no aparecen directamente vinculadas a estos, sino que responden más bien a las características del arreglo político existente en el país. El programa de gobierno de Sebastián Piñera (2018 - 2022) plantea su no renovación a 10 años de la ratificación.

10 El modelo de efectos combinados plantea de un modo algo más esquemático las siguientes hipótesis que condicionarían el impacto en las políticas públicas: "la presencia de aliados poderosos en la arena institucional o la opinión pública o por ambos factores simultáneamente" y "la naturaleza de las reivindicaciones: cuanto más conflictivas las reivindicaciones y más centrales las áreas de política en juego, menores las probabilidades del movimiento de obtener éxito" (Giugni 1998, citado por Tatagiba y Teixeira 2016: 87) 
uso de la consulta popular (que fue apoyada con recursos de Oxfam UK).

Otro aspecto relevante de considerar es el carácter multiescalar y las diversas formas de (des)articulación entre las escalas de acción (y los actores que en ellas operan). Especial importancia adquiere en las posibles consecuencias de los conflictos la variable internacional, por varias razones. En ocasiones algunas de ellas favorecen la actuación de las coaliciones locales frente a las políticas de los gobiernos. Ello se evidencia en el caso de las industrias extractivas, que son privilegiadas como fuente de ingresos por los estados, que ejercen presión sobre las comunidades locales para garantizar su desarrollo. ${ }^{11}$ Son múltiples los ejemplos donde se ha acudido a instancias internacionales con el fin de moderar o condicionar la presión gubernamental. Pero, al mismo tiempo, los condicionamientos internacionales -como acuerdos de libre comercio, garantía a las inversiones, préstamos de los organismos multilaterales- también limitan las posibilidades políticas de transformación y son denunciadas por los actores en conflicto como la causa de los mismos. Es necesario profundizar en las vinculaciones entre el activismo transnacional (Von Bülow 2010) y los conflictos socio-territoriales, en la medida que las políticas públicas se formulan, difunden y generalizan cada vez más en el marco de redes de políticas de carácter internacional.

A la justificación de la inversión extranjera directa como fuente de ingresos utilizada por los gobiernos neoliberales, se ha sumado la del "neoextractivismo" de los gobiernos de izquierda, que plantean la utilidad de la industria extractiva para financiar el desarrollo nacional y la redistribución de ingresos (Gudynas 2012).

\section{El cambio sobre los actores sociales y la acción colectiva en los territorios}

¿Cómo impactan estos conflictos a la estructuración de actores sociales y políticos en los territorios y a su capacidad de acción en los mismos? Una primera constatación es que estos movimientos locales no se articulan fácilmente en organizaciones nacionales que defiendan sus intereses de manera unificada.

Sin embargo surgen otro tipo de actores, muchas veces con el apoyo de la cooperación internacional. Este es un factor que muchas veces promueve la constitución de redes de actores locales en algunas áreas, sobre todo en materias ambientales, que están muy presentes en la agenda internacional. Los conflictos activan la presencia de estos actores, que tienen la ventaja de contar con mayor autonomía de acción respecto de autoridades locales y empresas. Sin embargo cuando estas articulaciones están demasiado afincadas en proyectos de ese tipo, sufren los vaivenes propios de la cooperación y no son sustentables. Lo mismo ocurre con muchas ONG locales cuyo financiamiento proviene de fuentes internacionales. Eso les da relativa independencia frente a los gobiernos, pero su continuidad se ve muchas veces amenazada por los cambios de las políticas de cooperación. Adicionalmente ese factor hace que las ONG dependientes de ese financiamiento, requieran funcionar en sintonía con esas agendas, lo cual no siempre coincide con las orientaciones de los actores locales del conflicto. En estos casos el fortalecimiento organizacional y de las redes no es sustentable.

Donde se advierten cambios, a partir de los estudios de caso, es en los marcos interpretativos (framing) que van adoptando 
los actores respecto de estos conflictos, que no se limita ya exclusivamente al problema específico que originó el conflicto, que puede resolverse con alguna compensación concreta. Como los conflictos son diferentes entre sí, no se trata de un marco idéntico para todos, pero van surgiendo críticas al modelo extractivo, normalmente en nombre de la defensa de los derechos humanos y la vida, frente a un modelo productivo centrado exclusivamente en la obtención de ganancias y la sobreexplotación de los recursos. En el caso de los conflictos en torno al agua, por ejemplo, este framing difiere sensiblemente de uno delimitado exclusivamente por la posesión de un recurso escaso (Yacoub, Duarte y Boelens 2015). El marco interpretativo que va surgiendo indica un discurso mucho más universalista y eventualmente interpelador para otros sectores no directamente afectados, lo cual es un componente relevante para la politización de estos movimientos. Desde los ecologistas de clase media proclives a estilos de vida "alternativos" a pequeños productores susceptibles de sufrir el mismo tipo de problemas y sectores de la Iglesia Católica interesados en propuestas socialmente inclusivas y que limiten el poder del dinero como factor de organización social. ${ }^{12}$ Kunrath et al. (2017) han expuesto las variantes que pueden producirse a partir de un framing compartido, a partir de las distinciones elaboradas inicialmente por Goffman y retomadas por los trabajos de David Snow que las aplican a los movimientos sociales. Es importante considerar que para estos autores, estas variantes no responden

En Perú el discurso de defensa de la vida y los derechos ha sido recogido por sectores católicos con influencias de la teología de la liberación; en Chile el obispo Luis Infanti de la región de Aysén ha desarrollado una verdadera pastoral del agua, convirtiéndose en protagonista del conflicto en Aysén. Para un análisis más amplio del papel de los centros jesuitas en estas materias, ver Coronado (2016). exclusivamente a decisiones estratégicas de los actores, como tendían a plantear tanto las teorías de movilización de recursos como las del proceso político (Idem. 153).

Algo similar sucede con conflictos en los que se defiende un determinado territorio en nombre del "patrimonio natural" que este representa, frente a las iniciativas de inversión y utilización productiva del mismo. Es decir se rechaza su intervención no por alguna consecuencia o externalidad negativa, sino porsu valorsimbólico, paisajístico o de aporte a la biodiversidad. En los casos donde hay participación de comunidades indígenas se verifica allí una confluencia entre las reivindicaciones territoriales de los indígenas y las orientaciones de los grupos ambientalistas, dando origen a coaliciones más amplias, por la naturaleza de los motivos que los movilizan (Delamaza et al 2017). ${ }^{13}$

Un último ámbito de impacto que interesa explorar y en torno al cual es necesario construir hipótesis teóricas acerca de cómo ocurre y de qué depende, es el impacto político local y regional. Es decir, aquello que podríamos considerar más genuinamente como impacto en los actores del territorio. Esto incluye tanto los propios actores sociales -si surgen nuevos, si se modifica el mapa de actores- como de los actores de la política local, es decir si emergen nuevos liderazgos o se modifica el mapa político electoral en el territorio. Donde más se aprecia una vinculación entre conflicto local y actores del territorio es en Perú. Allí durante el mismo período que se han intensificado los conflictos en torno a la minería, se han erigido nuevos liderazgos locales y regionales. Muchos de ellos han levantado

Para análisis de estos temas en el ámbito socio ambiental urbano, ver Santibáñez (2019). 
plataformas relacionadas precisamente con la defensa de sus localidades (la agricultura, el agua) que les han conferido gran apoyo electoral. Un caso proyectado al nivel nacional fue el de Marcos Arana, sacerdote y activista ambiental de Cajamarca, actividad que luego lo lleva a organizar un movimiento político (Tierra y Libertad) y a postular como precandidato presidencial en 2015 (Panfichi y Coronel, 2015). Pero es también el caso de varios dirigentes regionales que se han constituido plataformas políticas relativas a estos conflictos. En Chile, Iván Fuentes, dirigente sindical y líder del conflicto de Aysén en febrero de 2012, fue electo en 2013 como diputado por la Democracia Cristiana. ${ }^{14}$ En el caso del líder lafkenche, Adolfo Millabur, este fue electo alcalde de la comuna de Tirúa en varias ocasiones, pero posteriormente no logró acceder al congreso. Como fenómeno más general, una primera observación de los resultados de las elecciones municipales chilena de octubre de 2016 no revela una presencia extendida de los liderazgos provenientes de los movimientos locales en los nuevos municipios, aunque sí pérdida de apoyo de autoridades locales y modificaciones en el panorama político municipal.

Faltando analizar más casos en Bolivia y Colombia, nos inclinamos a pensar que este tránsito a la política local es altamente contingente al contexto político e institucional de cada país: la fortaleza del sistema de partidos, el tipo de partidos existentes y el grado y características de la descentralización. Un grado mayor de descentralización política y un sistema de partidos débil y desarticulado,

Luego de revelarse que su candidatura recibió financiamiento por parte de empresas pesqueras, no intentó volver a ser candidato por su región, desvinculándose en los hechos del movimiento social. Su intento en otro territorio, terminó en derrota electoral en 2017. provee oportunidades políticas mayores a los líderes locales, aunque estos no necesariamente se consoliden en el tiempo.

\section{Conclusiones}

El estudio de los conflictos socio-territoriales se puede abordar a partir de la teoría de los movimientos sociales, pero asumiendo que esa noción está experimentando una flexibilización y ampliación respecto de su acepción predominante. Se aprecia un panorama de actores más diverso y con menor peso organizativo, pero que al mismo tiempo ejerce mayores roles de mediación, roles que anteriormente eran cumplidos por organizaciones formales, como los partidos políticos. En el caso de los contextos rurales en América Latina -en especial en la Región Andinaeste proceso se ha visto acentuado a partir de los conflictos suscitados principalmente por el auge del extractivismo y la ampliación de la frontera minera y de otras industrias de explotación de los recursos naturales. Conflictos surgidos y arraigados en los territorios se proyectan también en el ámbito político.

La investigación sobre conflictos es más abundante en estudios de caso que en los resultados agregados del conjunto del fenómeno. Se observa una importante diversidad en los casos empíricos, pero aun así es posible establecer algunas regularidades desde el punto de vista de las consecuencias que ellos producen. El estudio de las consecuencias resulta un enfoque relevante para entender los cambios en las relaciones entre la acción colectiva y la dinámica política. No es posible establecer causalidad directa entre los conflictos socio-territoriales y los cambios políticos, pero 
es posible identificar el tipo de cambios en diferentes esferas y vincularlo con algunas de las características de los conflictos mismos.

La vinculación referida entre conflictividad socioterritorial y consecuencias políticas en ocasiones se produce a partir de un conflicto en particular, pero demaneramás extendidatiende a producirse a partir de los efectos acumulativos de pautas de conflicto que se diseminan o "contaminan" entre sí, así como también de los "efectos combinados". La diseminación se produce en parte debido a la multiescalaridad de los conflictos, que se expresa en la participación de diversos actores institucionales y no institucionales, cercanos o lejanos a los territorios donde se originan estos. También ella evoluciona según la combinación con estructuras de oportunidad o evolución de variables políticas por fuera del territorio, incluida la política empresarial. La dimensión internacional resulta especialmente relevante en este sentido.

Los casos indican consecuencias observables de importancia en el ámbito de las políticas públicas, así como en el cambio de relaciones entre los actores políticos locales. Menor impacto se aprecia en términos de cambios en el sistema político y el desarrollo de organizaciones nacionales, lo cual indica un tipo de politización diferente al pasado de la región. Por ello es que los cambios en políticas públicas aparecen como más frecuentes -hasta ahora- en los sectores de políticas menos articuladas con los arreglos políticos globales. Esto se aprecia en particular en aquellos arreglos que sustentan el extractivismo en sus diferentes versiones.

En cuanto a las consecuencias en el ámbito local, estos se revelan muy relacionados con los contextos institucionales y de características de la estructura del sistema político, por lo que no es posible hasta el momento establecer regularidades válidas, pues las consecuencias tienen diferente direccionalidad y sentido. Es un campo que requiere más investigación todavía. La investigación realizada abre interesantes pistas de investigación para estudiar las transformaciones de la acción colectiva y los vínculos con la dinámica política, a partir del surgimiento de actores de nuevo tipo, nuevas formas de expresión y que se expande a nuevos territorios en la región.

\section{Bibliografía}

Aguilera, O. y Álvarez, J. 2017. El ciclo de movilización en Chile 2005-2012: Fundamentos y proyecciones de una politización. Revista Austral de Ciencias Sociales, 29: 5-32. ISSN 0718-1795. Disponible en: <http://revistas.uach.cl/index.php/racs/article/ view/874>. Fecha de acceso: 20 nov. 2018.

Amenta, E. 2014. How to analyze the influence of movements. Contemporary Sociology (43): 16 -29.

Amenta, E. et al. 2010. The political consequences of social movements. Annu. Rev. Sociol.(36): 287-307.

Arce, M. 2015. La extracción de recursos naturales y la protesta social en el Perú. Lima: Fondo Editorial de la Pontificia Universidad Católica del Perú.

Arriagada, E. 2012. El conflicto de polimetales en Arica.
Movilización social, desarticulación local e intervención centralizada. En: Delamaza, G., Cunill, N. y Joignant, A. Nueva agenda de descentralización en Chile. Sentando más actores a la mesa. Santiago: RIL Editores / Universidad de Los Lagos, pp. 459-486.

Barros, V. 2013. TIPNIS: ¿Un conflicto ambiental o de territorio? Revista Letras Verdes. http://plataformaenergetica.org/ content/9172

Bebbington, A. 2016. De los conflictos a las políticas. Conferencia en el Instituto de Asuntos Públicos, Universidad de Chile, Santiago de Chile, 22 de agosto.

Bebbington, A. y Bury, J. 2013.. Subterranean Struggle. New dynamics of mining, oil, and gas in Latin America. Austin: 
University of Texas Press.

Boelens, R., Cremers, L. y Zwarteveen, M. (eds.) 2011. Justicia Hídrica. Acumulación, conflicto y acción social. Lima: IEP / Fondo Editorial PUCP / Justicia Hídrica

Bosi, L., Giugni, M. y Uba, K. 2016. The consequences of social movements. Cambridge: Cambridge University Press.

Calderón, F. (2012) Diez tesis sobre el conflicto social en América Latina. Revista CEPAL 107: 7-30.

Coronado, S. 2016. Latin American Jesuit social centers and environmental justice: advocay and support to local communities and knowledge-building from below. Journal fo Jesuit Studies, 9 (4): 664-678.

Cortez, M. 2016. Conflictos socio-territoriales en Chile y su impacto en la agenda política: el caso de Pascua Lama. Tesis para optar al grado de Magíster de Política y Gobierno, Universidad de Chile.

De Echave, J. et al. 2009. Minería y conflicto social. Lima: CBC / CIPCA / IEP / CIES

De la Fuente, M. 2017. Dinámica de los conflictos territoriales y régimen político en Bolivia. Ponencia presentada al Seminario "Consecuencias de los Conflictos Socio-territoriales en América Latina". Lisboa, 6 y 7 de marzo 2017.

Delamaza, G., Maillet, A. y Martínez, C. 2017. Socio-territorial conflicts in Chile: Configuration and politicization (2005-2014)

European Review of Latin American and Caribbean Studies/ Revista Europea de Estudios Latinoamericanos y del Caribe 104: 23-46. DOI: http://doi.org/10.18352/erlacs

Delamaza, G., Robles, C. y Montecinos, E. 2011. Redes de política pública y agendas de participación ciudadana en el Chile post- transicional: ¿Desafiando la política o recreando sus límites?, Revista Gestión y Política Pública, XXI (1):. 45-86.

Delamaza G. y Flores, F. 2012. Estrategia política territorial y reconocimiento de derechos colectivos. Incidencia del movimiento Indígena en la ley que crea el Espacio Costero Marino de los Pueblos Originarios. En: M. Fernández y Salinas, J. (eds). Disputas Territoriales de Recursos Públicos. La Defensa de los Derechos Territoriales desde las Comunidades Locales en Chile y Latinoamérica: Experiencias y Lecciones. Santiago: CIC/ULA/RIL Editores, pp. 93-128.

Diani, M. 2015. Revisando el concepto de movimiento social. Encrucijadas. Revista Crítica de Ciencias Sociales 9: 1-14.

Donoso, S. y Von Bülow, M. 2017. Social movements in Chile. Organization, trajectories, and political consequences. Cambridge: Palgrave.

Fraser, N. 2007. "Transnationalizing the public sphere". Theory, Culture and Society, 24 (4): 7-30.

Giugni, M. 2004. Social protest and policy change. Lanham MD: Rownman and Littlefield.

Giugni, M., Bosi, L. y Uba, K. 2013. Outcomes of Social Movements and Protest Activities. Oxford Bibliographies in "Political Science". Oxford: Oxford University Press.

González, A. 2017. La Colosa: ¿del conflicto territorial a la transformación de las políticas mineras? Ponencia presentada al
Seminario "Consecuencias de los Conflictos Socio-territoriales en América Latina". Lisboa, 6 y 7 de marzo 2017.

Gudynas, E. 2012. Estado compensador y nuevos extractivismos Las ambivalencias del progresismo sudamericano. Nueva Sociedad 237:. 128-146.

Haarstad, H. (ed.) 2012. New political spaces in latin american natural resource governance. London/ New York: Palgrave Macmillan

INDH 2016. Mapa de conflictos socio ambientales en Chile 2015. Santiago: INDH.

Kolb, F. 2005. Protest and opportunities. The political outcomes of social movements. Frankfurt/New York: Campus Verlag

Kunrath, M. Coutinho, F. y Mazzilli, M. 2017. Interpretação e ação coletiva: o "enquadramento interpretativo" no estudo de movimentos sociais. Sociología e Politica 25 (61): 143-164.

Li, F. 2015. Unearthing Conflict. Durham and London: Duke University Press.

OCMAL, 2015. Conflictos mineros en América Latina. Extracción, saqueo y agresión. La situación en 2014. Lima: OCMAL.

Panfichi, A., y Coronel, O. 2011. Conflictos hídricos en el Perú 2006 - 2010. Una lectura panorámica. Boelens, R., L. Cremers y M. Zwarteveen (eds.) Justicia Hídrica. Acumulación, conflicto y acción social. Lima: IEP / Fondo Editorial PUCP / Justicia Hídrica, pp. 122-143.

Panfichi, A. y Coronel, O. 2015. Régimen político y conflicto social en el Perú. Henríquez, N., Conflicto social en los andes. Protestas en Perú y Bolivia. Lima: Fondo Editorial PUCP, pp. 1364.

Paredes, M. 2015. La glocalización de las protestas mineras y las lecciones de Tambogrande. Henríquez, N., Conflicto social en los andes. Protestas en Perú y Bolivia. Lima: Fondo Editorial PUCP, pp. 135-158.

Paredes, M. 2016. The glocalization of mining conflict. Cases from Peru. The extractive industries and society 3 (4): 1046-1057.

Penaglia, F., Valenzuela, E. y Basaure, L. 2016. Acciones colectivas territoriales en Chile, 2011-2013: de lo ambientalreivindicativo al autonomismo regionalista. EURE 42 (125): 225250.

Pérez, M. 2013. Movimiento social de Aysén. Un caso de análisis de incidencia ciudadana en la agenda política. Tesis para optar al grado de Magíster en Gestión y Políticas Públicas. Universidad de Chile Departamento de Ingeniería Industrial.

Quiroga, S. et al. 2012. Perfiles de la conflictividad social en Bolivia (2009 - 2011). Análisis multifactorial y perspectivas. La Paz: Fundación UNIR

Roa, T. y L. Navas, 2014. Extractivismos, conflictos y resistencias. Bogotá: CENSAT / Amigos de la Tierra.

Santibáñez, H. (2019) Conflictos socio-ambientales en el Área Metropolitana de Valparaíso. Revista Austral de Ciencias Sociales, 35: 261-281, ISSN 0718-1795. Disponible en: <http://revistas. uach.cl/index.php/racs/article/view/4206>. Fecha de acceso: 18 jan. 2019 
Silva, E. 2015. Social movements, protest, and policy. ERLACS (100), 27-39.

Silva, E. 2016. Patagonia without dams! lessons of a David vs. Goliath campaign. New Orleans. Paper presented at the ISSCEDLA conference on "The Political Economy of the Extractive Imperative in Latin America: Reducing poverty and inequality vs. ensuring inclusion and sustainability?" The Hague, April 10-11, 2015.

Svampa, M. 2013. "Consenso de los commodities" y lenguajes de valoración en América Latina. Nueva Sociedad, 244: 30-46.

Tatagiba, L. y Teixeira, A. 2016. Efeitos combinados de os movimentos de moradia sobre os programas habitacionais autogestionários. Sociología e Política 24 (58): 85-102.

Tarrow, S. 2011. Power in Movement (3rd Ed.) New York/
Cambridge: Cambridge University Press

Ulloa, A. y Coronado, S. (eds.) 2016. Extractivismos y posconflicto en Colombia: retos para la paz territorial. Bogotá: CINEP/ Universidad Nacional de Colombia.

Urkidi, L. y Walter, M. 2011. Dimensions of environmental justice in anti-gold mining movements in Latin America. Geoforum 42 (6): 683-695. http://www.sciencedirect.com/science/article/pii/ S0016718511000868

Valenzuela, E. 2015. Territorios rebeldes. autonomías versus presicracia centralista. Santiago de Chile: Ediciones Universidad Alberto Hurtado.

Von Bülow, M. 2010. Building transnational networks: Civil society and the politics of trade in the Americas. Nueva York: Cambridge University Press. 